\title{
Outcomes and costs of penetrating trauma injury in England and Wales
}

\author{
Michael C. Christensen ${ }^{a}{ }^{*}$, Tina G. Nielsen ${ }^{3}$, Saxon Ridley ${ }^{b}$, Fiona E. Lecky ${ }^{c}$, Stephen \\ Morris d \\ a Global Development, Novo Nordisk A/S, Krogshojvej 55, DK-2880 Bagsvcerd, Denmark \\ b Glan Clwyd Hospital, Rhyl, Denbighshire LL18 5UJ, UK \\ C Trauma Audit and Research Network, University of Manchester, Eccles Old Road, Salford M6 \\ 8HD, UK \\ d Health Economics Research Group, Brunei University, Uxbridge, Middlesex UB8 3PH, UK
}

${ }^{*}$ Corresponding author. Tel.: +45 44420557; Fax: +4544427390. E-mail address:

mcrc@novonordisk.com.

\begin{abstract}
Summary
Background: Penetrating trauma injury is generally associated with higher short-term mortality than blunt trauma, and results in substantial societal costs given the young age of those typically injured. Little information exists on the patient and treatment characteristics for penetrating trauma in England and Wales, and the acute outcomes and costs of care have not been documented and analysed in detail. Methods: Using the Trauma Audit Research Network (TARN) database, we examined patient records for persons aged 18+ years hospitalised for penetrating trauma injury between January 2000 and December 2005. Patients were stratified by injury severity score (ISS).

Results: 1365 patients were identified; $16 \%$ with ISS 1-8, 50\% ISS 9-15, 15\% ISS 1624, 16\% ISS $25-34$, and $4 \%$ with ISS 35-75. The median age was 30 years and $91 \%$ of patients were men. Over $90 \%$ of the injuries occurred in alleged assaults. Stabbings were the most common cause of injury (73\%), followed by shootings (19\%). Forty- seven percent were admitted to critical care for a median length of stay of 2 days; median total hospital length of stay was 7 days. Sixty-nine percent of patients underwent at least one surgical procedure. Eight percent of the patients died before discharge, with a mean time to death of 1.6 days (S.D. 4.0). Mortality ranged from $0 \%$ among patients with ISS 1-8 to 55\% in patients with ISS > 34. The mean hospital cost per patient was $£ 7983$, ranging from $£ 6035$ in patients with ISS 9-15 to $£ 16,438$ among patients with ISS > 34. Costs varied significantly by ISS, hospital mortality, cause and body region of injury.

Conclusion: The acute treatment costs of penetrating trauma injury in England and Wales vary by patient, injury and treatment characteristics. Measures designed to reduce the incidence and severity of penetrating trauma may result in significant hospital cost savings.
\end{abstract}

KEYWORDS: Penetrating trauma; Outcomes; Costs; Treatment patterns; England; Wales; United Kingdom

\section{Introduction}

Recent evaluations of survival trends after trauma in the United Kingdom (UK) indicate that little improvement has occurred since 1994.14 In order to significantly enhance standards of care, the Royal College of Surgeons and the British Orthopaedic Association have recommended a national system of trauma service based on geographic trauma systems for England, Wales and Northern Ireland. 25 This recommendation highlights the need for documenting the current clinical 
practice of trauma care in the UK, and the outcomes and costs associated with such care.

Penetrating trauma injury is a serious burden to societies worldwide. In 2004 in the United States 29,569 died as a result of firearm injury and 2799 died from stabbing injuries, and it has been estimated that more than 64,000 non-fatal penetrating trauma injuries are treated in U.S. hospital emergency departments each year.4,23 In the UK it is estimated that somewhere between 22,000 and 57,900 young people were victims of a knife-related crime in 2004. 5 Penetrating trauma primarily affects young men and incurs substantial costs, not only in terms of the direct costs of medical care, but also the societal losses related to permanent disability and premature death. 4

The epidemiology of penetrating trauma injury differs significantly between Europe and the United States, in particular in regards to the overall incidence of penetrating trauma and the proportion of firearm-related injury. ${ }^{12,15}$ Few European studies have so far specifically examined the outcomes and costs of acute care of penetrating trauma despite these differences in trauma epidemiology. One UK study has examined the patterns and costs of acute care for 181 patients with gunshot wounds at one Manchester hospital, and found the clinical and financial impact to be substantial. 8 Eighty-six percent of the patients admitted required surgery resulting in an mean length of hospital stay of 7 days and mean treatment costs of $£ 2698$. However, no studies have addressed the national burden of penetrating trauma in terms of either of these parameters.

Therefore, the aims of this paper are to investigate the acute treatment patterns, outcomes and costs to the National Health Service (NHS) of penetrating trauma injury in England and Wales.

\section{Methods}

\section{Data and variables}

Data for this study were taken from the Trauma Audit Research Network (TARN), which includes data from 50\% ( $n=121)$ of all hospitals receiving trauma patients in England and Wales.27 TARN collects data on patients who sustain injuries resulting in immediate admission to hospital for $>3$ days, admission to Intensive Care Unit (ICU) or high dependency unit (HDU, intermediate intensive care), or death within 93 days. TARN is a voluntary national comparative audit of patients' outcomes and processes of care following admission to hospital with severe trauma. According to the National Centre for Health Outcomes Development, an agency under the National Health Service, the eligible population for TARN appears representative of the severe trauma population in England and Wales. 20 Between 90 and $97 \%$ of all eligible patients are recorded in the registry. TARN is supported by the Healthcare Commission without specific patient informed consent requirements or ethical approval because no patient identifiers are retained by TARN electronically or on paper and because TARN data is used for benchmarking the quality of hospital trauma care. Data for this study came exclusively from the TARN database without author access to patient records. TARN has Section 60 Patient Information Advisory Group approval; as this study involves the secondary analysis of TARN and other published data, ethics approval was not sought.

Data from TARN have been used in published studies on a range of topics including trends in trauma care, 14 the effect of neurosurgical care on head injury outcome, 24 outcome prediction in trauma, ${ }^{3}$ costs and outcomes in traumatic brain injury, ${ }^{19}$ and acute hospitalisation costs in blunt trauma. ${ }^{6}$ This is the first study using TARN data to investigate the acute treatment costs of penetrating trauma patients.

We included all patients hospitalised in TARN hospitals for penetrating trauma injuries between 1st January 2000 and 31st December 2005. The hospital completes a data entry sheet for each patient with information on age, gender, cause and type of injuries, severity of injuries, treatment provided at the scene of incident, en route to hospital, in Accident \& Emergency (A\&E), and any other care received at the hospital, including diagnostic tests, surgical procedures, and length of stay. Records of patients transferred between hospitals are linked so that full data for these patients are available. 
In our study, injury severity was measured using the injury severity score (ISS). ${ }^{2}$ The ISS is the standard measure of injury severity after trauma and it is based on an ordinal scale ranging from 1 to 75 , derived from the Abbreviated Injury Scale (AIS). Each AIS injury code is allocated an AIS score ranging from 1 (minor injury) to 6 (virtually unsurvivable injury). To compute the ISS, AIS injury codes are grouped into six body regions (head or neck, face, chest, abdominal or pelvic, extremities, and external) and the ISS is calculated as the sum of the squares of the highest AIS scores for the three most severely injured body regions. AIS codes of 6 are automatically allocated an ISS of 75 . As no definitive grouping of ISS scores exists, 26 we used the following categories: 1-8, 9-15, 1624, 25-34 and 35-75. In addition, we used data on: gender; age; cause and level of intent of injury; earliest recorded Glasgow Coma Score (GCS); systolic blood pressure and pulse rate in the A\&E Department; type and number of injuries sustained; discharge status; and year of admission to describe the patients treated.

We also used data from TARN on the characteristics of the treatment received. This included mode of arrival at hospital, time from emergency call to arrival at A\&E (when by ambulance), time spent in A\&E, highest grade and specialty of doctor attending the patient in A\&E, surgical procedures, admission to critical care, and length of stay. We also used data on whether or not the treating hospital had a neurosurgical centre and/or a spinal injury unit as a rough indicator of specialisation in trauma care. We excluded patients younger than 18 years of age at the time of injury from the analysis.

\section{Measuring costs}

To estimate costs we took the perspective of the NHS in England and Wales, i.e., only costs to the NHS were included. We focused on the costs of the treatment received during the initial hospitalisation. We calculated treatment costs for each patient based on the cost components likely to be the main drivers of cost during the initial hospitalisation period: cost of transportation (ambulance, helicopter), cost of hospital stay (A\&E, critical care, general ward), and cost of all surgical procedures performed. Resource use for every component was recorded for each patient in TARN using the data entry sheet. For all resource use we assigned unit costs obtained from external sources to each item (see Appendix A).

\section{Analysis}

First, we examined the demographic and clinical characteristics of the study sample and the acute treatment provided across groups of patients stratified by ISS. We stratified by ISS to examine how these characteristics varied by injury severity. We then computed treatment costs for every patient and calculated mean treatment costs by patient, injury and treatment characteristics and ISS group. We also computed hospital mortality rates for the same ISS groups.

Second, to identify the significant determinants of acute treatment costs we undertook multivariate regression analysis. We regressed individual level treatment costs against patient, injury and treatment characteristics. In the analysis, we excluded ' the variables that were used in the construction of the treatment cost variable. The variables included in the final regression models were selected using forward stepwise and backward stepwise selection procedures where the significance level for removal from the model was $p>0.01$, and significance level for addition to the model was $p<0.005$. We controlled for variations in provider practice by including hospital dummy indicator variables to control for the hospital in which the patient was treated (43 indicators). In the regression models we report robust standard errors that control for clustering within individuals and the cost model is estimated using least squares.

Some of the data collected by TARN and used in the analysis were missing in a number of patients. The main variables with missing data were time from emergency call to arrival at A\&E and surgical procedures. The latter may be missing because the relevant section on the data entry sheet completed by TARN participants describing the operative procedures received by each patient is completed by free text and may have been left blank. We report the numbers of 
observations used to calculate every statistic. When we calculated the acute care costs for each patient we did so using only patients with complete data.

\section{Results}

\section{Sample characteristics}

We identified 1365 patients with penetrating trauma injuries corresponding to $3.7 \%$ of the total number of TARN records in the 6-year study period.

\begin{tabular}{|c|c|c|c|c|c|c|c|}
\hline \multicolumn{8}{|c|}{ Table 1 Sample characteristics } \\
\hline & ISS & & & & & & \\
\hline & $1-8$ & $9-15$ & $16-24$ & $25-34$ & & $35-75$ & All \\
\hline$N$ & 213 & 678 & 206 & 219 & & 49 & 1,365 \\
\hline Male (\%) & 91.1 & 91.3 & 90.3 & 91.8 & & 81.6 & 90.8 \\
\hline $\begin{array}{c}\text { Age (yr), } \\
\text { median (IQR) }\end{array}$ & $31(23-42)$ & $\begin{array}{l}29(23- \\
38)\end{array}$ & $29(23-38)$ & \multicolumn{2}{|c|}{$31(22-40)$} & $34(27-44)$ & $\begin{array}{c}30(23 \\
39)\end{array}$ \\
\hline \multicolumn{8}{|c|}{ Age group (\%) } \\
\hline $18-24$ years & 31.9 & 35.8 & 35.0 & 33.8 & & 20.4 & 34.2 \\
\hline $25-44$ years & 50.2 & 50.2 & 49.0 & 48.0 & & 55.1 & 49.8 \\
\hline $45-64$ years & 14.1 & 12.0 & 14.6 & 14.6 & & 22.5 & 13.5 \\
\hline $65+$ years & 3.8 & 2.1 & 1.5 & 3.7 & & 2.0 & 2.5 \\
\hline \multicolumn{8}{|c|}{ Cause of injury (\%) } \\
\hline Vehicle collision & 1.9 & 1.0 & 2.9 & 1.8 & & 12.2 & 2.0 \\
\hline Falls & 2.4 & 1.0 & 3.4 & 2.3 & & 2.0 & 1.8 \\
\hline Shooting & 16.4 & 15.0 & 20.4 & 30.6 & & 16.3 & 18.6 \\
\hline Stabbing & 66.2 & 80.1 & 71.8 & 61.2 & & 67.4 & 73.2 \\
\hline Other & 13.2 & 2.8 & 1.5 & 4.1 & * & 2.0 & 4.4 \\
\hline \multicolumn{8}{|c|}{ Level of intent (\%) } \\
\hline Non-intentional & 17.1 & 4.1 & 7.8 & 5.3 & & 18.2 & 7.2 \\
\hline Alleged assault & 79.8 & 94.6 & 92.3 & 94.0 & & 81.8 & 91.6 \\
\hline Other & 3.1 & 1.3 & 0.0 & 0.7 & & 0.0 & 1.2 \\
\hline $\mathrm{n}$ & 129 & 459 & 129 & 150 & & 33 & 900 \\
\hline $3-5$ & 0.5 & 1.7 & 12.2 & 23.1 & & 39.5 & 7.6 \\
\hline $6-8$ & 0.5 & 0.6 & 2.1 & 4.3 & & 11.6 & 1.8 \\
\hline $9-12$ & 0.5 & 2.3 & 3.7 & 6.5 & & 9.3 & 3.1 \\
\hline
\end{tabular}




\begin{tabular}{|c|c|c|c|c|c|c|c|c|}
\hline $13-15$ & 98.4 & 95.3 & 81.9 & & 66.1 & & 39.5 & 87.5 \\
\hline $\mathrm{n}$ & 190 & 644 & 188 & & 186 & & 43 & 1251 \\
\hline $\begin{array}{r}\text { Systolic bloo } \\
\text { in A\&E ( }\end{array}$ & $\begin{array}{l}\text { pressure } \\
\mathrm{mHg})\end{array}$ & & & & & & & \\
\hline Mean $(95 \% \mathrm{Cl})$ & $\begin{array}{c}133(130- \\
137)\end{array}$ & $\begin{array}{l}126 \\
(124- \\
128)\end{array}$ & $\begin{array}{l}119 \\
(114\end{array}$ & 124) & $\begin{array}{l}116 \\
(111\end{array}$ & $\begin{array}{c}- \\
121)\end{array}$ & $\begin{array}{l}107(94- \\
119)\end{array}$ & $\begin{array}{l}124(122 \\
-126)\end{array}$ \\
\hline $\mathrm{n}$ & 180 & 605 & 170 & & 169 & & 34 & 1158 \\
\hline \multicolumn{9}{|c|}{$\begin{array}{l}\text { Injuries by body region with AIS } \\
3+(\%)\end{array}$} \\
\hline Mean $(95 \% \mathrm{Cl})$ & $90(87-93)$ & $\begin{array}{l}92(90- \\
94)\end{array}$ & $96(92$ & $\begin{array}{c}- \\
100)\end{array}$ & 99 (95 & 103) & $\begin{array}{c}103(93- \\
112)\end{array}$ & $\begin{array}{l}94(92- \\
95)\end{array}$ \\
\hline $\mathrm{n}$ & 181 & 624 & 172 & & 176 & & 31 & 1184 \\
\hline \multicolumn{9}{|c|}{$\begin{array}{l}\text { Injuries by body region with AIS } \\
\qquad 3+(\%)\end{array}$} \\
\hline Head & 0.0 & 3.4 & 15.5 & & 27.9 & & 36.7 & 9.8 \\
\hline Face & 0.0 & 1.8 & 1.0 & & 2.3 & & 8.2 & 1.7 \\
\hline Neck & 0.0 & 3.4 & 7.8 & & 4.6 & & 8.2 & 3.9 \\
\hline Thorax & 0.0 & 49.4 & 48.1 & & 57.5 & & 77.6 & 43.8 \\
\hline Abdomen & 0.0 & 21.2 & 35.4 & & 22.8 & & 38.8 & 21.0 \\
\hline Spine & 0.0 & 0.9 & 2.4 & & 7.8 & & 10.2 & 2.4 \\
\hline Upper limb & 0.0 & 9.0 & 5.8 & & 5.5 & & 10.2 & 6.6 \\
\hline Lower limb & 0.0 & 10.2 & 10.2 & & 5.9 & & 14.3 & 8.1 \\
\hline Mean & 0.0 & 1.2 & 2.1 & & 2.5 & & 4.0 & 1.5 \\
\hline $\begin{array}{l}\text { Mortality \% (95\% } \\
\mathrm{Cl})\end{array}$ & $0.0(0.0-0.0)$ & $\begin{array}{l}1.8(0.8- \\
2.8) \\
\end{array}$ & $\begin{array}{r}12.1 \\
16\end{array}$ & & $\begin{array}{r}22.4( \\
27 \\
\end{array}$ & & $\begin{array}{l}55.1(40.7- \\
69.5) \\
\end{array}$ & $\begin{array}{c}8.3(6.8- \\
9.7)\end{array}$ \\
\hline
\end{tabular}

Notes: The sample size is equal to the total number in each ISS group $\mathrm{n}$ other than where indicated by $\mathrm{n}$.

Two hundred and thirteen patients (16\%) had an ISS of 1-8, $678(50 \%)$ had an ISSof 9-15, 206 (15\%) had an ISS of 16-24, 219 (16\%) had an ISS of 25-34, and $49(4 \%)$ had an ISS of 35-75 (Table 1). Ninety-one percent of the patients were male, and the median age was 30 years (IQR 23-39 years).

The main cause of penetrating injury was stabbing (73.2\%), followed by shooting $(18.6 \%)$. There was no significant year on year change in these proportions (stabbings vs. shootings). Motor vehicle

collisions and falls accounted for around $2 \%$ of all penetrating injuries. Over $90 \%$ of injuries were alleged assaults. The median (interquartile range [IQR]) GCS was 15(15-15) across the whole sample, falling to 8 (3-14) in the ISS 35-75 group. Mean (95\% confidence interval [Cl]) systolic blood pressure in A\&E was $124 \mathrm{mmHg}(122-126)$ and mean pulse rate was 94 beats per minute (92-95). 
The most common serious injuries (with an AIS score of 3 or more) were to the thorax (44\%), abdomen (21\%) and head (10\%). The average patient sustained 1.5 injuries with an AIS score of 3 or more, ranging from 0 in the ISS 1-8 group to 4 in the ISS 35-75 group.

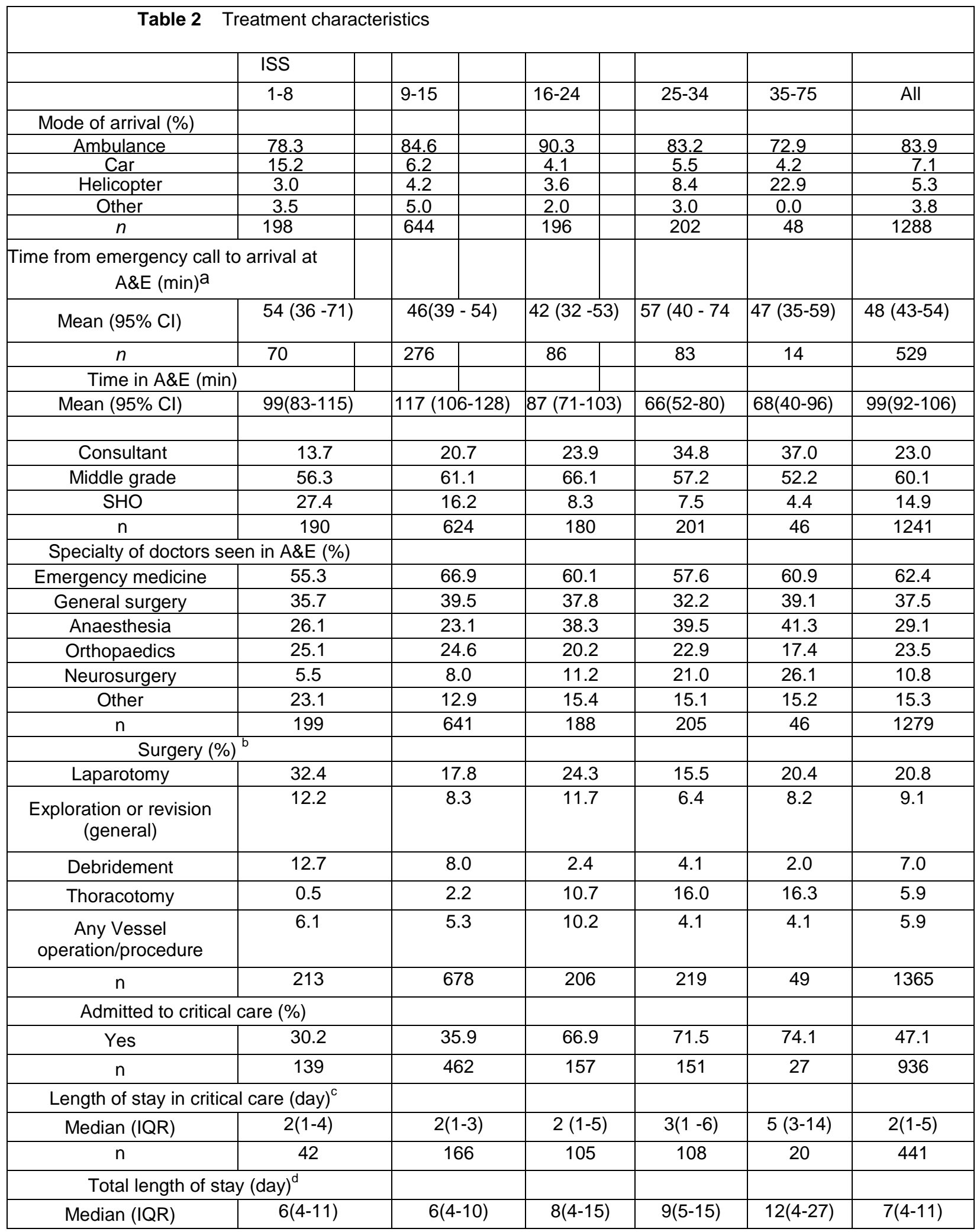




\begin{tabular}{|c|c|c|c|c|c|c|}
\hline $\mathrm{n}$ & 139 & 462 & 157 & 151 & 27 & 936 \\
\hline \multicolumn{2}{|c|}{ Hospital has a neurosurgical centre (\%) } & & & & & \\
\hline Yes & 41.8 & 48.5 & 51.5 & 57.5 & 69.4 & 50.1 \\
\hline $\mathrm{n}$ & 213 & 678 & 206 & 219 & 49 & 1365 \\
\hline \multicolumn{2}{|c|}{ Hospital has a spinal injuries unit (\%) } & & & & & \\
\hline Yes & 6.1 & 2.5 & 4.4 & 1.8 & 2.0 & 3.2 \\
\hline $\mathrm{n}$ & 213 & 678 & 206 & 219 & 49 & 1365 \\
\hline
\end{tabular}

a Among those arriving by ambulance.

b Results are reported for procedures undergone by at least $5 \%$ of all patients, ranked by \% for All.

C Among those who were admitted to critical care.

$d$ Including length of stay in critical care. The figures pertain both to those who were admitted to critical care and those who were not.

\begin{tabular}{|c|c|c|c|c|c|c|}
\hline \multicolumn{7}{|c|}{ Table 3 Hospital mortality (\%) by sample characteristics } \\
\hline & ISS & & & & & \\
\hline & $1-8$ & $9-15$ & $16-24$ & $25-34$ & $35-75$ & All \\
\hline Hospital mortality (\%) & 0.0 & 1.8 & 12.1 & 22.4 & 55.1 & 8.3 \\
\hline$n$ & 213 & 678 & 206 & 219 & 49 & 1365 \\
\hline Hospital mortality \{\%) & & & & & & \\
\hline By gender & & & & & & \\
\hline Male & 0.0 & 1.8 & 11.8 & 22.4 & 55.0 & 8.1 \\
\hline Female & 0.0 & 1.7 & 15.0 & 22.2 & 55.6 & 10.4 \\
\hline By age group & & & & & & \\
\hline 18-24 years & 0.0 & 1.6 & 9.7 & 17.6 & 50.0 & 6.2 \\
\hline 25-44 years & 0.0 & 1.8 & 11.9 & 21.9 & 51.9 & 8.1 \\
\hline 45-64 years & 0.0 & 2.5 & 16.7 & 21.9 & 63.6 & 11.4 \\
\hline 65+ years & 0.0 & 0.0 & 33.3 & 75.0 & 100.0 & 23.5 \\
\hline By cause of injury & & & & & & \\
\hline Vehicle collision & 0.0 & 0.0 & 0.0 & 25.0 & 83.3 & 22.2 \\
\hline Falls & 0.0 & 0.0 & 28.6 & 0.0 & 0.0 & 8.0 \\
\hline Shooting & 0.0 & 0.0 & 9.5 & 26.9 & 62.5 & 10.6 \\
\hline Stabbing & 0.0 & 1.8 & 12.8 & 20.1 & 48.5 & 7.2 \\
\hline Other & 0.0 & 10.5 & 0.0 & 33.3 & 100.0 & 10.0 \\
\hline By level of intent & & & & & & \\
\hline Non-intentional & 0.0 & 0.0 & 20.0 & 12.5 & 83.3 & 12.3 \\
\hline Alleged assault & 0.0 & 1.6 & 14.3 & 23.4 & 63.0 & 9.0 \\
\hline Other & 0.0 & 16.7 & & 0.0 & & 9.1 \\
\hline By Glasgow Coma Score group & 0.0 & 45.5 & 73.9 & 69.8 & 100.0 & 72.6 \\
\hline 3-5 & 0.0 & 0.0 & 0.0 & 25.0 & 80.0 & 27.3 \\
\hline 6-8 & 0.0 & 0.0 & 14.3 & 16.7 & 0.0 & 7.7 \\
\hline 9-12 & 0.0 & 0.7 & 2.6 & 4.9 & 23.5 & 1.6 \\
\hline 13-15 & & & & & & \\
\hline \multicolumn{2}{|c|}{} & & & & \\
\hline
\end{tabular}




\begin{tabular}{|c|c|c|c|c|c|c|}
\hline \multicolumn{7}{|c|}{ By systolic blood pressure in A\&E group } \\
\hline$<100 \mathrm{mmHg}$ & 0.0 & 1.2 & 17.5 & 24.4 & 53.3 & 14.0 \\
\hline $100-119 \mathrm{mmHg}$ & 0.0 & 0.8 & 0.0 & 9.8 & 22.2 & 2.8 \\
\hline $120-139 \mathrm{mmHg}$ & 0.0 & 0.9 & 5.9 & 10.8 & 20.0 & 2.7 \\
\hline $140-159 \mathrm{mmHg}$ & 0.0 & 0.0 & 3.0 & 13.8 & 0.0 & 2.2 \\
\hline $160+m m H g$ & 0.0 & 0.0 & 0.0 & 11.8 & 66.7 & 3.4 \\
\hline \multicolumn{7}{|c|}{ By pulse rate in A\&E group } \\
\hline <80b.p.m. & 0.0 & 1.8 & 7.7 & 19.0 & 50.0 & 5.5 \\
\hline 80-99 b.p.m. & 0.0 & 0.4 & 3.2 & 12.8 & 33.3 & 2.6 \\
\hline 100-119 b.p.m. & 0.0 & 0.7 & 6.5 & 11.6 & 18.2 & 3.8 \\
\hline 120+b.p.m. & 0.0 & 1.4 & 7.7 & 20.5 & 50.0 & 9.4 \\
\hline \multicolumn{7}{|c|}{ By injuries by body region with AIS $3+$} \\
\hline Head & & 0.0 & 9.4 & 31.1 & 66.7 & 25.4 \\
\hline Face & & 0.0 & 0.0 & 0.0 & 75.0 & 13.0 \\
\hline Neck & & 8.7 & 12.5 & 20.0 & 50.0 & 15.1 \\
\hline Thorax & & 1.8 & 10.1 & 16.7 & 50.0 & 9.4 \\
\hline Abdomen & & 0.0 & 17.8 & 16.0 & 52.6 & 10.8 \\
\hline Spine & & 0.0 & 0.0 & 0.0 & 0.0 & 0.0 \\
\hline Upper limb & & 1.6 & 0.0 & 8.3 & 40.0 & 4.4 \\
\hline Lower limb & & 1.4 & 14.3 & 15.4 & $57 \mathrm{~A}$ & 9.1 \\
\hline
\end{tabular}

Notes: Numbers in italics are based on 10 or fewer observations.

\begin{tabular}{|c|c|c|c|c|c|c|}
\hline & & & & & & \\
\hline & $1-8$ & $9-15$ & $16-24$ & $25-34$ & $35-75$ & All \\
\hline \multicolumn{7}{|c|}{ Total cost $(£)$} \\
\hline $\begin{array}{l}\text { Mean (95\% } \\
\mathrm{Cl})\end{array}$ & $\begin{array}{c}6,501(5,540- \\
7,463)\end{array}$ & $\begin{array}{c}6,053(5,487- \\
6,583)\end{array}$ & $\begin{array}{c}9,453(8,072- \\
10,833)\end{array}$ & $\begin{array}{c}12,347(9,831- \\
14,862)\end{array}$ & $\begin{array}{c}16,438(10,384- \\
22,493)\end{array}$ & \begin{tabular}{|c}
$7,983(7,381$ \\
$8,584)$
\end{tabular} \\
\hline $\begin{array}{l}\text { Median } \\
\text { (IQR) }\end{array}$ & $\begin{array}{c}4,728(3,524- \\
7,181)\end{array}$ & \begin{tabular}{|c}
$4,721(2,727-$ \\
$7,279)$
\end{tabular} & $\begin{array}{c}7,218(4,336- \\
11,739)\end{array}$ & $\begin{array}{c}7,376(4,328- \\
14,227)\end{array}$ & $\begin{array}{c}10,773(6,043- \\
26,864)\end{array}$ & $\begin{array}{c}5,473(3,293- \\
8,835)\end{array}$ \\
\hline Range & $551-36,904$ & $551-70,100$ & 711- 53,038 & $551-107,533$ & $816-64,374$ & $551-107,533$ \\
\hline $\mathrm{n}$ & 130 & 443 & 150 & 139 & 27 & 889 \\
\hline \multicolumn{7}{|c|}{ Mean total cost $(£)$} \\
\hline \multicolumn{7}{|l|}{ By gender } \\
\hline Male & 6,558 & 5,997 & 9,398 & 12,180 & 18,673 & 7,965 \\
\hline Female & 6,035 & 6,421 & 10,030 & 14,108 & 8,618 & 8,150 \\
\hline \multicolumn{7}{|c|}{ By age group } \\
\hline $18-24$ & 5,338 & 4,878 & 8,560 & 12,994 & 23,314 & 7,119 \\
\hline $25-44$ & 6,400 & 6,630 & 9,362 & 10,630 & 15,935 & 7,962 \\
\hline $45-64$ & 10,296 & 6,497 & 12,387 & 14,038 & 15,570 & 10,052 \\
\hline
\end{tabular}




\begin{tabular}{|c|c|c|c|c|c|c|}
\hline $65+$ years & 6,483 & 8,412 & 5,146 & 36,234 & 2,201 & 9,775 \\
\hline \multicolumn{7}{|c|}{ By cause of injury } \\
\hline \multicolumn{2}{|c|}{ Vehicle collision 9,596 } & 8,962 & 22,496 & 69,432 & 10,541 & 16,185 \\
\hline Falls & 5,211 & 4,729 & 10,895 & 18,615 & 43,196 & 10,740 \\
\hline Shooting & 6,977 & 9,961 & 10,249 & 13,039 & 9,091 & 10,307 \\
\hline Stabbing & 6,088 & 5,441 & 8,683 & 11,237 & 16,706 & 7,196 \\
\hline Other & 7,369 & 5,895 & 12,977 & 17,371 & & 8,488 \\
\hline \multicolumn{7}{|c|}{ By level of intent } \\
\hline Non- & 7,046 & 7,788 & 21,024 & 37,035 & 10,541 & 12,644 \\
\hline Alleged & 6,357 & 6,034 & 8,569 & 12,033 & 14,814 & 7,699 \\
\hline Other & 3,667 & 3,229 & & & & 3,448 \\
\hline \multicolumn{7}{|c|}{ By Glasgow Coma Score group } \\
\hline $3-5$ & 36,904 & 14,240 & 9,407 & 15,306 & 5,040 & 13,040 \\
\hline $6-8$ & & 13,543 & 13,664 & 11,184 & 5,969 & 9,679 \\
\hline $9-12$ & 5,430 & 13,639 & 18,924 & 19,792 & 22,743 & 17,756 \\
\hline $13-15$ & 5,697 & 5,681 & 9,048 & 9,857 & 17,112 & 6,926 \\
\hline \multicolumn{7}{|c|}{ By injuries by body region with AIS 3+ } \\
\hline Head & & 4,399 & 5,953 & 12,702 & 14,125 & 9,431 \\
\hline Face & & 5,769 & 4,449 & 16,940 & 11,727 & 9,461 \\
\hline Neck & & 7,607 & 12,042 & 18,130 & 14,102 & 10,955 \\
\hline Thorax & & 4,589 & 10,368 & 13,497 & 17,462 & 8,232 \\
\hline Abdomen & & 8,122 & 10,533 & 14,180 & 19,411 & 10,535 \\
\hline Spine & & 4,411 & 6,006 & 13,706 & 36,639 & 14,696 \\
\hline Upper limb & & 6,687 & 9,987 & 26,557 & 15,885 & 10,240 \\
\hline Lower limb & & 9,157 & 8,024 & 24,618 & 21,459 & 12,191 \\
\hline \multicolumn{7}{|c|}{ By discharge status } \\
\hline Alive & 6,501 & 6,043 & 9,743 & 12,911 & 20,859 & 8,094 \\
\hline Dead & & 2,528 & 4,915 & 4,201 & 3,808 & 4,278 \\
\hline
\end{tabular}

Notes: Numbers in italics are based on 10 or fewer observations.

\begin{tabular}{|c|c|c|c|c|c|c|}
\hline \multicolumn{2}{|c|}{ Table 5 Mean treatment costs by treatment characteristics* } & & \\
\hline & ISS & & & & & \\
\hline & $1-8$ & $9-15$ & $16-24$ & $25-24$ & $35-75$ & All \\
\hline Mean total cost (£) & & & & & & \\
\hline By mode of arrival & & & & & & \\
\hline Ambulance & 7,004 & 5,886 & 9,619 & 12,861 & 16,781 & 8,072 \\
\hline Helicopter & 6,725 & 7,078 & 6,704 & 10,156 & 15,754 & 9,045 \\
\hline Car & 3,841 & 5,767 & 8,369 & 6,185 & & 5,450 \\
\hline Other & 7,622 & 7,587 & 9,938 & 15,475 & & 8,761 \\
\hline
\end{tabular}




\begin{tabular}{|c|c|c|c|c|c|c|}
\hline \multicolumn{7}{|c|}{ By grade of most senior doctor seen in $A \& E$} \\
\hline Consultant & 7,330 & 5,352 & 9,517 & 12,746 & 12,252 & 8,324 \\
\hline Middle grade & 6,244 & 6,277 & 9,207 & 12,103 & 19,286 & 8,001 \\
\hline $\mathrm{SHO}$ & 6,782 & 5,504 & 7,709 & 7,522 & 10,773 & 6,325 \\
\hline \multicolumn{7}{|c|}{ By specialty of doctors seen in $A \& E$} \\
\hline Emergency medicine & 5,819 & 5,655 & 8,311 & 9,739 & 19,065 & 7,039 \\
\hline General surgery & 5,694 & 6,182 & 9,941 & 11,361 & 15,779 & 7,699 \\
\hline Anaesthesia & 7,040 & 7,636 & 12,541 & 11,993 & 20,931 & 10,072 \\
\hline Orthopaedics & 6,013 & 6,482 & 11,903 & 17,343 & 16,539 & 9,248 \\
\hline Neurosurgery & 6,681 & 5,295 & 6,389 & 7,228 & 17,083 & 7,163 \\
\hline Other & 8,871 & 6,023 & 7,932 & 22,126 & 14,664 & 9,297 \\
\hline \multicolumn{7}{|l|}{ By surgery ${ }^{3}$} \\
\hline Laparotomy & 5,979 & 9,058 & 10,918 & 15,496 & 30,032 & 10,197 \\
\hline Exploration or revision & 5,678 & 6,608 & 11,744 & 19,869 & 17,290 & 9,645 \\
\hline Debridement & 9,580 & 7,316 & 10,148 & 22,054 & 7,060 & 9,706 \\
\hline Thoracotomy & & 9,356 & 13,013 & 16,777 & 21,916 & 14,493 \\
\hline Any vessel operation/procedure & 6,997 & 7,390 & 9,377 & 10,341 & 18,879 & 8,546 \\
\hline \multicolumn{7}{|c|}{ By length of stay in critical care (day) } \\
\hline 0 & 4,474 & 4,170 & 4,581 & 3,513 & 4,601 & 4,221 \\
\hline $1-5$ & 8,858 & 7,831 & 8,410 & 9,161 & 9,704 & 8,410 \\
\hline $6-10$ & 18,175 & 14,972 & 16,828 & 20,993 & 20,160 & 17,904 \\
\hline $11+$ & 27,177 & 42,123 & 35,761 & 49,067 & 40,735 & 41,755 \\
\hline \multicolumn{7}{|c|}{ By total length of stay (day) ${ }^{b}$} \\
\hline $0-5$ & 3,776 & 3,206 & 4,118 & 3,525 & 3,808 & 3,491 \\
\hline $6-10$ & 5,729 & 5,868 & 7,599 & 7,192 & 9,861 & 6,353 \\
\hline $11-20$ & 10,282 & 10,050 & 10,910 & 11,745 & 13,495 & 10,808 \\
\hline $21-30$ & 17,763 & 11,781 & 19,503 & 17,790 & 27,955 & 17,196 \\
\hline $31-40$ & 13,700 & 14,024 & 22,597 & 27,539 & 26,864 & 20,319 \\
\hline $41-50$ & 23,175 & 19,065 & 28,779 & 20,844 & 37,981 & 26,031 \\
\hline $51+$ & 36,904 & 33,190 & 35,899 & 46,115 & 53,785 & 41,378 \\
\hline \multicolumn{7}{|c|}{ By whether or not hospital has a neurosurgical centre } \\
\hline No & 7,643 & 7,442 & 10,987 & 14,660 & 17,604 & 9,314 \\
\hline Yes & 5,610 & 5,116 & 8,280 & 11,006 & 16,174 & 7,106 \\
\hline \multicolumn{7}{|c|}{$\begin{array}{l}\text { By whether or not hospital has a spinal } \\
\text { injuries unit }\end{array}$} \\
\hline No & 6,542 & 6,092 & 9,328 & 12,490 & 16,438 & 8,033 \\
\hline Yes & 5,210 & 4,140 & 11,995 & 2,551 & & 6,297 \\
\hline
\end{tabular}

Notes: Numbers in italics are based on 10 or fewer observations. ${ }^{1}$ Among those arriving by ambulance.

$\mathrm{a}$ Results are reported for procedures undergone by at least $5 \%$ of all patients only.

$\mathrm{b}$ Including length of stay in critical care. The figures pertain both to those who were admitted to critical care and those who were not. 


\section{Treatment characteristics}

Eighty-four percent of patients arrived at the hospital by ambulance, $7 \%$ by car, and $5 \%$ by helicopter (Table 2). Among those traveling by ambulance, mean time from emergency call to arrival at the A\&E Department was 48 min (95\% Cl 43-54), and $87 \%$ arrived within one hour of the call. The mean time in the A\&E Department was 99 min $(95 \% \mathrm{Cl} 92106)$. Twenty-three percent of the patients were seen by a consultant in A\&E, $60 \%$ were seen by a middle grade doctor (defined as senior registrar, registrar, specialist registrar with up to 5 years of experience, staff grade, associate specialist and research fellow) and $15 \%$ were seen by a senior house officer (SHO).

Sixty-nine percent of the patients underwent at least one surgical procedure; the most common were laparotomy (21\%), general surgical exploration (9\%), wound debridement (7\%), thoracotomy (6\%) and vessel-related procedures (5\%).

Forty-seven percent of the patients were admitted to critical care. The percentage ranged from $30 \%$ in the ISS 1 -8 group to $74 \%$ in the ISS $35-75$ group. The low critical care admission rate among those with ISS $35-75$ is due to the early mortality in this group: the mean $(95 \% \mathrm{Cl})$ and median (IQR) times to death after arrival at A\&E among those with ISS 35-75 who died were 1.0 days (-0.1-2.1) and 0 (0-0) days, respectively. The median (IQR) length of stay in critical care among those who were admitted was 2 days (1-5). Median (IQR) total length of stay, including length of stay on a general ward and in critical care, was 7 days (4-11).

\section{Hospital mortality}

The overall mortality rate among the 1365 penetrating trauma patients was $8.3 \%$. Of the hospital deaths observed, the mean time to death was 1.5 days ( $95 \% \mathrm{Cl} 0.8-2.2)$. Mean time to death was 2.3 days (-1.5-6.1) for those injured in motor vehicle collisions, 17.0 days (4.3-29.7) for falls, 0.6 days (0.2-1.1) for shootings, 1.0 day (0.2-1.8) for stabbings, and 4.7 days (0.4-8.9) for other causes of injury.

Hospital mortality by patient characteristics is reported in Table 3. Mortality rates were higher in females and increased with age. Overall, mortality was higher in those injured by shooting (11\%) than by stabbing (7\%), yet the highest mortality was incurred in the motor vehicle accident category (22\%). Unintentional penetrating injuries had higher mortality rates than alleged assaults. Across the whole sample mortality increased as GCS declined. Most types of serious injury (AIS3+) were associated with higher mortality with the exception of spinal and upper limb injuries.

\section{Treatment costs}

The mean total NHS hospital cost per patient during the initial hospitalisation was $£ 7983(95 \% \mathrm{Cl}$ $£ 73818584$ ) (Table 4). Treatment costs were highest among patients injured in a motor vehicle collision, followed by falls and firearm injuries. Treatment costs were markedly higher among those injured unintentionally. There was no clear relationship between GCS and costs. Every type of serious injury were associated with higher on treatment costs. In all ISS groups mortality 
had a negative effect on treatment costs. Across all patients, length of stay in a general ward accounted for the largest percentage of costs (37\%), followed by length of stay in critical care (32\%). The next most important component was surgical procedures (23\%), followed by travel to the A\&E Department (4\%) and A\&E costs (3\%).

The impact of treatment characteristics on treatment costs is shown in Table 5. Treatment costs varied by mode of hospital arrival (helicopter was the most expensive). Across all patients mean treatment costs were higher if the patient was seen by a consultant in A\&E, and if the patient was seen by an anaesthetist. Patients who were seen by a consultant had more severe injuries than those who were not seen by a consultant, which probably explains the higher costs incurred by this group (mean ISS 17.2 vs. 13.6, mean difference 3.6, 95\% Cl 2.0-5.1). Patients treated by an anaesthetist were also more severely injured than those who did not see doctors from this specialty (mean ISS 16.7 vs. 13.4 , mean difference $3.3,95 \% \mathrm{Cl} 1.9-4.8$ ). Unsurprisingly, treatment costs were positively correlated with length of stay in critical care and total length of stay.

\section{Multivariate analysis of costs}

The results of the multivariate regression analysis are in Table 6. In the treatment cost model the covariates explain $23 \%$ of the variation in hospital costs. The most significant determinants of costs were ISS (positively correlated with costs), and hospital mortality (negatively correlated). Patients with an ISS score in the range $35-75$ had on average $£ 12,500$ higher costs than those with an ISS score in the range 1-15, and patients who died in hospital had on average ${ }^{1} £ 9300$ lower treatment costs than those who survived. Other statistically significant determinants of treatment costs were serious injuries of the abdomen (which were positively correlated with costs), cause of injury (stabbing) and admission to a hospital with a neurosurgical centre (both of which were negatively correlated).

\begin{tabular}{|l|r|r|c|}
\hline \multicolumn{4}{|c|}{ Table 6 Multivariate analysis of mean treatment costs } \\
\hline & Treatment costs & & \\
\hline & Coefficient & $t$ & $P$ \\
\hline ISS $^{\mathrm{a}}$ & & & \\
\hline $16-24$ & 3197 & 4.4 & $<0.001$ \\
\hline $25-34$ & 5647 & 4.8 & $<0.001$ \\
\hline $35-75$ & 12,463 & 4.6 & $<0.001$ \\
\hline Discharge status: dead & -9285 & -6.0 & $<0.001$ \\
\hline Injuries by body region with AIS 3+: abdomen & 2271 & 3.0 & 0.003 \\
\hline Cause of injury: stabbing & -2414 & -3.4 & 0.001 \\
\hline Hospital with neurosurgical centre & -3237 & -3.1 & 0.002 \\
\hline Observations & 889 & & \\
\hline Adjusted $R^{2}$ & 0.232 & & \\
\hline
\end{tabular}

The model also includes 43 hospital indicators. The coefficient is the mean change in treatment costs associated with each variable, controlling for the other variables in the model.

a The omitted category is 1-15. 


\section{Discussion}

This study provides a detailed description of the demographic and clinical characteristics of penetrating trauma patients in England and Wales, their causes of injury, acute treatment received and outcomes in terms of mortality. It is the first study to provide a detailed assessment of the NHS hospital costs associated with penetrating trauma injury in England and Wales.

The patient characteristics, outcomes and costs of blunt trauma in England and Wales have previously been described. ${ }^{6}$ Using this research it is possible to compare point estimates for patient characteristics, outcomes and costs for blunt trauma patients to those for penetrating trauma patients. The most notable differences in patient characteristics for penetrating trauma appear to be the following: penetrating trauma patients are on average 16 years younger than blunt trauma patients and a substantially larger proportion are men (91\% vs. 63\%). In accordance with previous findings, ${ }^{7,16}$ the hospital mortality from penetrating trauma was slightly higher (8\% vs. $7 \%$ ), and time to death from hospital admission was substantially shorter (1.6 days vs. 9.3 days). Penetrating trauma patients had shorter time from emergency call to arrival in A\&E than blunt trauma (48.3 $\mathrm{min}$ vs. $70.7 \mathrm{~min}$ ), possibly due to the fact that penetrating trauma primarily occur in urban areas and hence closer to the treating hospitals. In addition transport time to hospital in blunt trauma patients injured in road traffic collisions may be delayed by the time spent on extrication at the scene of accident. Penetrating trauma patients also spent only half the time on average in A\&E (99.2 min vs. $193.8 \mathrm{~min}$ ), probably because assessment and disposal is usually more straightforward than in multisystem blunt trauma. A greater proportion of penetrating trauma patients were admitted to critical care ( $47 \%$ vs. $29 \%)$, but for a shorter length of stay (median 2 days vs. 4 days). The overall hospital length of stay was also shorter for penetrating trauma (median 7 days vs. 9 days). The mean initial

hospitalisation cost associated with penetrating trauma was lower than for blunt trauma ( $£ 7983$ vs. $£ 9530$ per patient). Thus, there appear to be differences in patient characteristics, outcomes and costs between blunt trauma patients and penetrating trauma patients. It is important to note, however, that the comparison is based on point estimates only; the differences may not be statistically significant.

A number of studies have characterised the outcomes and treatment patterns of penetrating trauma in the United States. ${ }^{1}$ The latest data from the American College of Surgeons National Trauma Data Bank, covering 640 trauma centres, found an overall mortality rate of $9.6 \%$ for penetrating trauma and a cause-specific mortality rate of $15.4 \%$ for gunshot wounds and $1.6 \%$ for cuts/piercings. ${ }^{22}$ The mean length of hospital stay associated with these specific types of penetrating trauma was 6.5 and 3.2 days, respectively, in 2001-2005, whilst the overall mean length of stay for the entire cohort was 5.1 days. The specific inclusion criteria in the TARN registry of admission for 3 days or more prevents a direct comparison on outcomes, length of stay and hospital costs. Interestingly Mock et al and Klein et al, comparing costs and outcomes for patients with gunshot wounds to stabbing wounds in the United States, found the cost of shooting to be higher than stabbing; a finding similar to our observation. ${ }^{13,17}$ Miller and Cohen, providing a summary of cost estimates for the acute care of penetrating trauma in the United States, found mean charges of US $\$ 11,002$; again an estimate not substantially different from our study. ${ }^{15}$ Only one study has been conducted on the cost of penetrating trauma in the United Kingdom. Cowey et al. reviewed the cost of 187 gunshot injuries treated a teaching hospital in Manchester from 
1995 to 2000 and found a mean cost of $£ 2698$ for patients admitted for in-patient care based on local unit cost data. ${ }^{8}$ In our study we found the acute care cost of gunshot wounds to be $£ 10,307$. The difference is again likely to be explained by the requirement of admission among survivors for 3 days or more in the TARN registry, which precludes inexpensive short stay cases.

As seen in our study intentional violence is the major cause of penetrating trauma in England and Wales, and it is associated with substantial treatment costs even in the short term. Though the costs of penetrating trauma resulting from shooting is higher than from stabbing (as seen in other countries ${ }^{15}$ ), the most commonly used weapon in violent crime in England and Wales is actually a knife. Recent data from the Centre for Crime and Justice Studies at King's College suggest that between 22,000 and 57,900 young people could have been victims of a knife-related crime in $2004 .^{5}$ At an average cost of $£ 7699$ per penetrating injury from alleged assault and a total of 417 injuries per year requiring hospitalisation for at least 3 days (assuming TARN captures data for $50 \%$ of all trauma injuries in the United Kingdom), the total acute care cost of this type of injury alone may exceed $£ 3.2$ million annually. Considering the additional medical costs of rehabilitation, and the broader societal costs resulting from lost productivity, permanent disability, premature death and the pain and suffering by the victims and their families, this money could be better spent on prevention strategies that reduce violent incidents. For instance, since October 2007, the laws on selling knives changed in England and Wales and it became illegal for shopkeepers to sell knives to anyone under the age of $18 .^{11}$ Since May 2004 the Home Office has disbursed more than $£ 1.25$ million in grants to local community groups to support knife intervention projects in England and Wales. ${ }^{11}$ We acknowledge a number of limitations with the current study. First, we did not include treatment cost occurring after the initial hospitalisation period, including cost of rehabilitation, home care support and any subsequent hospitalisations related to penetrating trauma. Second, the retrospective nature of the study implies reliance on the quality and completeness of the data reported to the TARN database. We observed incomplete data on a number of important treatment parameters, particularly the time from emergency call to arrival at A\&E and surgical procedures. If these data, particularly those relating to surgical procedures, are not missing at random across the different patient characteristics recorded, this could bias our results. Third, whilst there is strong evidence that the hospitals participating in TARN can be considered nationally representative in regards to the patients treated for severe trauma, ${ }^{20}$ the registry is still based on voluntary participation. A possibility of bias therefore does exist insofar as non-participating hospitals may treat patients who are systematically different from those included in our study. Fourth, we excluded patients who died before they reached the hospital and our estimates relate only to patients hospitalised for penetrating trauma for 3 days or more. Fifth, the indirect costs related to lost productivity and time spent away from other activities, as well as the costs associated with the pain and suffering by victims and relatives were not included. The cost of follow up care and the indirect costs can be substantial, and in fact represent the majority of the lifetime societal costs of penetrating trauma. ${ }^{5}$ Sixth, the unit costs used in the analysis are by necessity crude. In particular, the unit costs of hospital stays are not specific to penetrating trauma patients. Clearly, it would be more appropriate to use the actual costs incurred by each patient included in the analysis, yet these data were not available in TARN for this analysis.

In summary, our study provides detailed information on patient characteristics, treatment patterns, mortality and costs of penetrating trauma in the acute-care setting in England and 
Wales. Our findings indicate that the initial hospital costs associated with penetrating trauma are substantial, and vary to a considerable degree by patient, injury and treatment characteristics. Public health initiatives that aim to reduce the incidence and severity of penetrating trauma are therefore likely to produce significant savings in acute trauma care costs.

\section{Acknowledgements}

The authors would like to thank Omar Bouamra and Tom Jenks at the Trauma Audit Research Network for providing the data used in this study and gratefully acknowledge the huge effort made by individual clinicians and hospitals in collecting and submitting raw data to the TARN database. TARN is funded by its participating hospitals.

\section{Appendix A. Unit costs}

\begin{tabular}{|c|c|c|c|}
\hline Cost Component & Unit & $\begin{array}{l}\text { Unit Cost } \\
(£)\end{array}$ & Source and Notes \\
\hline \multicolumn{4}{|l|}{$\begin{array}{l}\text { Mode of arrival at } \\
\text { hospital }\end{array}$} \\
\hline Ambulance & Cost per minute & 5.50 & \multirow{2}{*}{$\begin{array}{l}\text { Curtis and Netten } 9 \text { (p.112) Cost per minute of } \\
\text { emergency ambulance service London Air } \\
\text { Ambulance Website } \\
\text { http://www.londonsairambulance.com/ Mean cost } \\
\text { per mission }\end{array}$} \\
\hline Helicopter & $\begin{array}{l}\text { Mean cost per } \\
\text { patient journey }\end{array}$ & 1650 & \\
\hline \multicolumn{4}{|l|}{ Hospital stay } \\
\hline A\&E Department & $\begin{array}{l}\text { Mean cost per } \\
\text { attender }\end{array}$ & 278 & $\begin{array}{l}\text { Department of Health }{ }^{10} \text { Mean cost per attender } \\
\text { across all A\&E Healthcare Resource Groups }\end{array}$ \\
\hline General Ward & $\begin{array}{l}\text { Mean cost per } \\
\text { day }\end{array}$ & 281 & $\begin{array}{l}\text { Department of Health } 10 \text { Mean national average } \\
\text { unit cost per day across all non-elective } \\
\text { Healthcare Resource Groups }\end{array}$ \\
\hline Critical Care Unit & $\begin{array}{l}\text { Mean cost per } \\
\text { day }\end{array}$ & 1328 & $\begin{array}{l}\text { Department of Health }{ }^{10} \text { Mean cost per day in } \\
\text { Intensive Care Unit/Intensive Therapy Unit }\end{array}$ \\
\hline $\begin{array}{l}\text { Surgical } \\
\text { procedures }^{a}\end{array}$ & Duration (min) & $\begin{array}{l}\text { Unit Cost } \\
\text { (E) }\end{array}$ & Source and Notes \\
\hline Laparotomy & 160 & 1810 & \multirow{6}{*}{$\begin{array}{l}\text { TARN; NICE }{ }^{21} \text { The duration in minutes for each } \\
\text { procedure was computed internally using the } \\
\text { TARN database. The unit costs were then } \\
\text { computed by multiplying the duration by the } \\
\text { variable cost per minute from NICE }{ }^{21} \text { and adding a } \\
\text { fixed cost per procedure also taken from NICE. } \\
\text { This method has been used in previous UK cost } \\
\text { analyses of trauma care } 18\end{array}$} \\
\hline $\begin{array}{l}\text { Exploration or revision } \\
\text { (general) }\end{array}$ & 139 & 1675 & \\
\hline Debridement & 139 & 1680 & \\
\hline Thoracotomy & 157 & 1790 & \\
\hline $\begin{array}{l}\text { Any vessel } \\
\text { operation/procedure }\end{array}$ & 182 & 1951 & \\
\hline Suture & 125 & 1591 & \\
\hline
\end{tabular}

a Details are reported in the table for in the analysis. The duration and unit procedures undergone by at least $5 \%$ of penetrating trauma patients. 103 procedures are included cost range from 10 min and £852 for Gastroscopy to 475 min and £3828 for Escharatomy. 


\section{Conflict of interest}

This study was funded by Novo Nordisk A/S. M. Christensen and T. Nielsen are employees of Novo Nordisk A/S. S. Morris has received consultancy fees and S. Ridley has received honoraria from Novo Nordisk Ltd. TARN received an unrestricted grant for making data available for this study.

\section{References}

1) Arbabi S, Jurkovich GJ, Wahl WL, et al. A comparison of prehospital and hospital data in trauma patients. J Trauma 2004 56:1029-32.

2) Baker SP, O'Neill B, Haddon W, et al. The injury severity score: a method for describing patients with multiple injuries and evaluating emergency care. J Trauma 1974 14:187-96.

3) Bouamra O, Wrotchford A, Hollis S, et al. A new approach to outcome prediction in trauma: a comparison with the TRISS model. J Trauma 2006 61:701-10.

4) Centers for Disease Control and Prevention. Morbidity and mortality weekly report. JAMA 2000 283:47-8.

5) Centre for Crime and Justice Studies. Knife crime: ineffective reactions to a distracting problem? A review of evidence and policy; August 2006. Available on-line at: http://www.kcl.ac.uk/depsta/rel/ccjs/knife-crime-2006.pdf.

6) Christensen ME, Saxon R, Lecky F, et al. Cost of blunt trauma in England and Wales. Crit Care, in press.

7) Clark DE, Lucas FL, Ryan LM. Predicting hospital mortality, length of stay, and transfer to long-term care for injured patients. J Trauma 2007 62:592-600.

8) Cowey A, Mitchell P, Gregory J, et al. A review of 187 gunshot wound admissions to a teaching hospital over a 54-month period: training and service implications. Ann RColl Surg Engl 2004 86:104-7.

9) Curtis L, Netten A. Unit costs of health and social care 2004. University of Kent; 2004. Available on-line at: http://www.hems-london.org.uk/contents/FAQ.aspx.

10) Department of Health. NHS reference costs 2004. Appendix SRC1 -NHS Trust reference cost index. Department of Health 2005.

11) Home Office. Violent Crime. Available on-line at: http://www.homeoffice.gov.uk/crimevictims/reducing-crime/vio lent-crimel.

12) Kauvar DS, Wade DE. The epidemiology and modern management of traumatic hemorrhage: US and international perspectives. Crit Care 2005 9(Suppl. 5):S1-9.

13) Klein SR, Kanno IJ, Gilmore DA, Wilson SE. The socioeconomic impact of assault injuries on an urban trauma center. Am Surg 1991 57:793-7.

14) Lecky FE, Woodford M, Bouamra O, Yates DW. Lack of change in trauma care in England 
and Wales since 1994. Emerg Med J 2002 19:520-3.

15) Miller TR, Cohen MA. Costs of gunshot and cut/stab wounds in the United States, with some Canadian Comparisons. Accid Anal Prev 1997 29:329-34.

16) Millham FH, LaMorte WW. Factors associated with mortality in trauma: re-evaluation of the TRISS method using the National Trauma Data Bank. J Trauma 2004 56:1090-6.

17) Mock C, Plicher S, Maier R. Comparison of the costs of acute treatment for gunshot and stab wounds: further evidence of the need for firearms control. J Trauma 1994 36:516-21.

18) Morris S, Ridley S, Munro V, Christensen ME. Cost effectiveness of recombinant activated factor VII for the control of bleeding in patients with severe blunt trauma injuries in the United Kingdom. Anaesthesia 2007 62:43-52.

19) Morris S, Ridley S, Munro V, et al. Traumatic brain injury in England and Wales: treatment, outcomes and hospital costs. Anaesthesia, in press.

20) National Centre for Health Outcomes Development. Clinical and health outcomes knowledge database. Trauma Audit Research Network. Available on-line at http:// www.nchod.nhs.uk/NCHOD/DocDat 2.nsf/b14278ba7209e9ff882571 d800125c52/55d3f 326ddb8936b 6525725a002f9f6f!OpenDocument.

21) National Institute for Health and Clinical Excellence. Technology Appraisal Guidance 83 Laparoscopic surgery for inguinal hernia repair; September 2004. p. 13-4.

22) National Trauma Data Bank Report 2006, version 6.0. Available on-line at: www.facs.org/trauma/ntdb/ntdbannualre-port2006.pdf.

23) National Vital Statistics Reports, vol. 55, no. 19; August 21, 2007.

24) Trauma Audit \& Research Network. Trends in head injury outcome from 1989 to 2003 and the effect of neurosurgical care: an observational study. Lancet 2005 366: 1538-44.

25) Royal College of Surgeons of England. Better care for the severely injured. A joint report from the Royal College of Surgeons of England and the British Orthopaedic Association 2000.

26) Stevenson M, Segui-GomezM, Lesohier I, etal. An overview of the injury severity score and the new injury severity score. Inj Prev 2001 7:10-3.

27) The Trauma Audit and Research Network (TARN). Available on line at: http://www.tam.ac.uk. 